\title{
A multidisciplinary approach to treating low back pain in the ED: improving patient outcomes
}

\section{Lesley Beique, Jason Martyn}

Background: In collaboration with emergency physicians, the physiotherapy and pharmacy teams at the Rockyview General Hospital (RGH) implemented a novel, multidisciplinary, evidence-based pathway that functions by deploying a "rapid access back-care team” (RABT) to address low back pain (LBP) in urban emergency departments (EDs).

The pathway starts with having a physiotherapist (PT) conducting a neuromusculoskeletal exam, prior to the physician. They provide treatment and guidance including manual therapy, mobilization, education, home-exercises and referral to community resources. The pharmacist then reviews medications, discusses pain management, prescribes analgesia and creates a plan for outpatient analgesia. This occurs while the patient awaits the physician (an average wait time of 2 hours at our site), avoiding increases to length of stay (LOS) and reducing burden on physicians.

Implementation: To operationalize this pathway successfully, a site requires dedicated PT and pharmacy services in the ED, as the unpredictable timing of LBP referrals requires a regular presence of the RABT. The selected PT and pharmacist must be confident, outgoing practitioners with a solid understanding of LBP, red-flags, and appropriate treatment.

Our project team consisted of physiotherapists, pharmacists, nurses, physicians, managers, and QI leaders, formed to facilitate a collaborative approach to implementation. The Prosci ${ }^{\circledR}$ ADKAR model and Plan-Do-Study-Act (PDSA) cycles were used to implement the pathway and troubleshoot operational challenges.

Evaluation Methods: Front-line staff manually collected data on response time, treatments, adverse events, and resources provided. The investigators reviewed patient charts to record opioid prescriptions, DI referrals, and arrival/discharge times of the patients. We compared outcomes of patients seen by the RABT to historical site data of patients with a discharge diagnosis of LBP from the ED.

We actively sought feedback from physicians, nurses, and the leadership group to ensure that unintended consequences or near-misses were identified early on. We reviewed interim data such as LOS and average time-to-assessment, to identify areas for improvement. This data and feedback were addressed via bimonthly PDSA cycles. We also administered patient and staff satisfaction surveys before and after site implementation of the pathway to develop an understanding of patient and staff thoughts, feelings and experiences with the service model.

Results: We studied these outcomes in 44 patients exposed to our RABT. Patients who saw a physiotherapist prior to the physician had shorter median ED LOS (3.2h vs. 4.0h), lower diagnostic imaging rates (36.4\% vs. 49.4\%) and less opioid prescribing (31.8\% vs. 49.2\%). No patients returned to the ED within 72 hours post evaluation, compared to the 
7.6\% historical recidivism. Not all patients were seen by a pharmacist. When performing a subgroup analysis of patients seen by both a pharmacist and physiotherapist prior to physician, opioid prescriptions were found to drop significantly from a baseline of $49.2 \%$ to $16.7 \%$.

\section{Advice and Lessons Learned:}

1. Service hours are ideally aligned with higher patient demand times, but should ultimately be chosen to minimize service disruptions and maximize overlap between interdisciplinary members of the RABT team.

2. Regular PDSA cycles (every 2-4 weeks) are useful to review interim data and address operational issues that arise during implementation. This ensures the pathway evolves to fit the contextual needs of the site. Reviewing early results motivates the team, and discussing practice issues allows clinicians to identify where improvements can be made.

3. When this pathway was initially implemented, one unintended consequence was the increase in ED LOS for patients referred to the RABT following physician assessment. In addition, this subgroup did not show significant reductions in opioid prescriptions or DI referrals. Referrals were subsequently restricted to before the physician only and were ideally completed by the triage nurse to maximize time for the RABT. 